\title{
A COMPARISON OF MEASURED CRAB AND VELA GLITCH HEALING PARAMETERS WITH PREDICTIONS OF NEUTRON STAR MODELS
}

\author{
FRONEFIELD CRAWFORD \\ Department of Physics, Haverford College, Haverford, PA 19041; fcrawfor@haverford.edu \\ AND \\ MAREK DEMIAŃSKI \\ Institute of Theoretical Physics, University of Warsaw, Warsaw, Poland; and \\ Department of Astronomy, Williams College, Williamstown, MA 01267; \\ marek.demianski@fuw.edu.pl \\ Received 2003 March 11; accepted 2003 June 11
}

\begin{abstract}
There are currently two well-accepted models that explain how pulsars exhibit glitches, sudden changes in their regular rotational spin-down. According to the starquake model, the glitch healing parameter $Q$, which is measurable in some cases from pulsar timing, should be equal to the ratio of the moment of inertia of the superfluid core of a neutron star (NS) to its total moment of inertia. Measured values of the healing parameter from pulsar glitches can therefore be used in combination with realistic NS structure models as one test of the feasibility of the starquake model as a glitch mechanism. We have constructed NS models using seven representative equations of state of superdense matter to test whether starquakes can account for glitches observed in the Crab and Vela pulsars, for which the most extensive and accurate glitch data are available. We also present a compilation of all measured values of $Q$ for Crab and Vela glitches to date that have been separately published in the literature. We have computed the fractional core moment of inertia for stellar models covering a range of NS masses and find that for stable NSs in the realistic mass range $1.4 \pm 0.2$ $M_{\odot}$, the fraction is greater than 0.55 in all cases. This range is not consistent with the observational restriction $Q \lesssim 0.2$ for Vela if starquakes are the cause of its glitches. This confirms results of previous studies of the Vela pulsar that have suggested that starquakes are not a feasible mechanism for Vela glitches. The much larger values of $Q$ observed for Crab glitches $(Q \gtrsim 0.7)$ are consistent with starquake model predictions and support previous conclusions that starquakes can be the cause of Crab glitches.
\end{abstract}

Subject headings: pulsars: individual (PSR B0531+21, PSR B0833-45) — stars: neutron

\section{INTRODUCTION}

Soon after the discovery of radio pulsars (Hewish et al. 1968), they were identified as rotating, highly magnetized neutron stars (NSs; Gold 1968, 1969; Ostriker \& Gunn 1969). It was observationally established that pulsar periods slowly and monotonically increase with time as a result of magnetic braking and rotational energy loss. Early in 1969, it was noticed that the Vela pulsar suddenly increased its angular velocity (Reichley \& Downs 1969; Radhakrishnan \& Manchester 1969). This distinct and sudden increase in rotational frequency, known as a glitch, was later regularly observed in the Vela and Crab pulsars and, more infrequently, in other pulsars (e.g., Shemar \& Lyne 1996; Lyne, Shemar, \& Smith 2000). The Crab (PSR B0531+21) and Vela (PSR B0833-45) pulsars are the two best-studied glitching pulsars, since they have each been observed to glitch a number of times and are bright and easy to monitor. These pulsars have the most extensive and accurate glitch data published and currently provide the best test of the physical mechanisms by which pulsar glitches occur.

There are currently two competing models that are well accepted to explain how pulsars glitch. In simplest terms, both models treat the NS as a two-component body, with a superfluid interior core surrounded by a rigid external crust (e.g., Ruderman 1972). These two components are dynamically weakly coupled through the magnetic field.

In the starquake glitch model (Ruderman 1976; Baym \& Pines 1971; Alpar et al. 1996), a slight equatorial oblateness in the crust can be formed if the NS is born rapidly spinning. As the pulsar slows down via magnetic braking, the deformation cannot be supported through centrifugal pressure from the core, and the crust can suddenly crack under gravity. The subsequent reduction in oblateness reduces the moment of inertia, resulting in a sudden increase in the rotational frequency, seen as a glitch.

Alternatively, in the vortex unpinning model of glitches (Anderson \& Itoh 1975; Alpar et al. 1984, 1993), angular momentum is stored in vortices of superfluid that migrate outward from the core as the star slows down. These vortices can become pinned to nuclei in the transition region between the core and outer crust, thereby preventing angular momentum transfer to the crust. A differential rotation develops between the core and crust until a catastrophic unpinning of the vortices occurs. Angular momentum is suddenly transferred to the crust, spinning it up. Since the crust is tightly coupled to the external magnetic field, this sudden spin-up in the rotation period is observed as a glitch.

Within the starquake model, the time evolution of a pulsar's rotational frequency after the occurrence of a glitch (Wang et al. 2000) can be fitted by the equation

$$
\Omega(t)=\Omega_{0}(t)+\Delta \Omega_{t=0}\left[1-Q\left(1-e^{-t / \tau}\right)\right]+\Delta \dot{\Omega}_{p} t .
$$

Here, $\Omega_{0}(t)$ represents an extrapolation of the preglitch frequency evolution, $\Delta \Omega_{t=0}$ is the magnitude of the change in the rotational frequency at the time of the glitch $(t=0), \tau$ is a characteristic exponential healing or recovery time after 
the glitch, and $Q$ is the fraction of the initial frequency change that is eventually recovered (known as the healing parameter). The last term accounts for any permanent (not recovered) change $\Delta \dot{\Omega}_{p}$ in the frequency derivative after the glitch. The parameter $Q$ in equation (1) can be determined observationally (Shapiro \& Teukolsky 1983) from the jump in $\Omega$ and its first and second time derivatives:

$$
Q=\frac{\Delta \dot{\Omega}_{t=0}^{2}}{\Delta \ddot{\Omega}_{t=0} \Delta \Omega_{t=0}} .
$$

In the starquake model, $Q$ is related to the moments of inertia of the components of the star according to (Pines, Shaham, \& Ruderman 1974)

$$
Q=\frac{I_{\text {core }}}{I_{\text {total }}},
$$

where $I_{\text {core }}$ and $I_{\text {total }}$ are respectively the moment of inertia of the superfluid core and the moment of inertia of the entire star. We have constructed a series of models of the NS interior using representative high-density equations of state (EOSs) in order to test whether the calculated moments of inertia in the models satisfy the prediction of the starquake model for Crab and Vela glitches. We have compared the model results with the measured values of $Q$ from Crab and Vela glitches to see if they are consistent.

\section{EQUATIONS OF STATE}

We have constructed NS models using seven representative EOSs of superdense matter from which parameterized model stars can be produced. For densities $\rho \lesssim 1.6 \times 10^{14} \mathrm{~g}$ $\mathrm{cm}^{-3}$ (crustal densities), we have used three EOSs for three separate density regimes (see, e.g., Shapiro \& Teukolsky 1983). These three EOSs are described by Feynman, Metropolis, \& Teller (1949), Baym, Pethick, \& Sutherland (1971), and Baym, Bethe, \& Pethick (1971). The seven highdensity EOSs, which dominate the macroscopic characteristics of the model stars, are briefly described below. Further details about each EOS can be found in the sources referenced.

\subsection{The BJW Equation of State}

For densities above the nuclear density, a combination of EOSs presented by Canuto (1975) was used for the modeling. For densities up to $\sim 5 \times 10^{15} \mathrm{~g} \mathrm{~cm}^{-3}$, the EOS described by Bethe \& Johnson (1974) was employed. This EOS is an improvement on the work of Reid (1968), which includes a repulsive nucleonic core arising from the exchange of vector mesons in a hyperonic liquid. At higher densities, the EOS of Walecka (1974) was used, although the NS models produced in this density regime were beyond the stability limit. In this EOS, nucleons interact attractively via exchange of scalar mesons and repulsively via exchange of more massive vector mesons. Summaries of these EOSs are also presented by Shapiro \& Teukolsky (1983). We call this EOS BJW.

\subsection{The FPS Equation of State}

Lorenz, Ravenhall, \& Pethick (1993) refer to an EOS calculated by Friedman \& Pandharipande (1981) for highdensity neutron and nuclear matter. This EOS employs the microscopic V14 two-body potential, a three-nucleon inter- action (TNI) potential, and hypernetted chain techniques. This EOS was modified by fitting the microscopic interaction of Friedman \& Pandharipande to a Skyrme-like energy density function (Skyrme 1959). The essential feature of the Skyrme model is a two-body interaction that has the spatial character of a two-body delta function plus derivatives. We refer to this EOS as FPS.

\subsection{The GWM Equation of State}

This EOS is based on a variant of the theory of nuclear field coupling (Zimanyi \& Moszkowski 1990) in which the scalar field is coupled to the derivative of the nucleon field. A modification proposed by Glendenning, Weber, \& Moszkowski (1992), called the hybrid derivative coupling model, replaces the purely derivative coupling of the scalar field to baryons and vector mesons with a Yukawa point and derivative coupling to baryons and both vector fields. The coupling model is consistent with the experimentally inferred binding energy of lambda hyperons in nuclear matter. The resulting EOS, called GWM here, maintains equilibrium between all baryons and leptons to convergence. The existence of hyperons significantly softens this EOS.

\subsection{The HKP Equation of State}

This EOS, proposed by Haensel, Kutschera, \& Proszynski (1981), supposes that a minimal condition for the properties of high-density matter is that it must produce the observed values for the saturation properties of nuclear matter. The relativistic mean field theory of Serot (1979) is assumed in which nucleon interactions are governed by the exchange of neutral scalar and neutral vector mesons, pions, and rho mesons. This theory is an extension of the relativistic mean field theory of Walecka (1974). The authors assume a conservative range of possible nuclear saturation densities, the mean of which is $2.8 \times 10^{14} \mathrm{~g} \mathrm{~cm}^{-3}$. The EOS used here, called HKP, is based on this nuclear saturation value.

\subsection{The WFF Equation of State}

Wiringa, Fiks, \& Fabrocini (1988) describe a microscopic EOS of dense nuclear matter constrained by nucleonnucleon scattering data. The interaction includes a twonucleon Urbana $v_{14}$ potential and a TNI three-body potential term (Lagaris \& Pandharipande 1981a, 1981b). The three-body potential includes a repulsive term, the primary effect of which is a reduction in the intermediaterange attraction of the two-nucleon potential, and an attractive term, which becomes negligible at high densities. We refer to this EOS as WFF.

\subsection{The APR Equations of State}

Akmal, Pandharipande, \& Ravenhall (1998) describe a set of realistic EOSs based on the Argonne $v_{18}$ two-nucleon interaction (Wiringa, Stoks, \& Schiavilla 1995), calculated using variational chain summation methods. This is supplemented with a relativistic boost correction term (Forest, Pandharipande, \& Friar 1995) and a TNI term based on the Urbana IX model (Pudliner et al. 1995). The two cases of pure neutron matter (PNM) and symmetric nuclear matter (SNM) are separately considered. The latter is composed of equal numbers of neutrons and protons in beta equilibrium. The two EOSs used to construct the models presented here 
were derived from a parabolic fit to and extrapolation of the tabulated nucleon density and energy values given for PNM and SNM by Akmal et al. (1998). We refer to the two EOSs based on PNM and SNM as $\operatorname{APR}(p)$ and $\operatorname{APR}(s)$, respectively.

\section{THE MODELING PROCEDURE}

To construct parameterized NS models for each EOS, we first chose a central density $\rho_{c} \equiv \rho(0)$ for each stellar model. The EOS and the relativistic expressions for stellar structure (Oppenheimer \& Volkoff 1939) were used to calculate the pressure gradient and incremental mass contained in concentric shells as the model iterated outward from the center of the star. The relativistic structure equations are

$$
\begin{gathered}
\frac{d m(r)}{d r}=4 \pi r^{2} \rho(r), \\
\frac{d P(r)}{d r}=-\left[\rho(r)+\frac{P(r)}{c^{2}}\right] \frac{G\left[m(r)+4 \pi r^{3} P(r) / c^{2}\right]}{r^{2}\left[1-2 G m(r) / r c^{2}\right]},
\end{gathered}
$$

where $m(r)$ represents the mass of the star internal to a distance $r$ from the center of the star. When the pressure reaches zero, $P(R)=0$, the edge of the star is reached $(r=R)$, and the relevant macroscopic parameters can be read off.

To calculate the relativistic moment of inertia $I(r)$ interior to a radius $r$, expressions given by Arnett $\&$ Bowers (1977) were used that account for the Lense-Thirring framedragging effect (e.g., Glendenning 2000; see discussion below):

$$
\frac{d I(r)}{d r}=\frac{8 \pi}{3} \frac{\left[\rho(r)+P(r) / c^{2}\right] r^{4} e^{-\phi(r)}}{\left[1-2 G m(r) / r c^{2}\right]^{1 / 2}} \frac{\bar{\omega}(r)}{\Omega} .
$$

Here, $\Omega$ is the observed stellar rotational frequency, and $\bar{\omega}(r)$ is the angular velocity of the star at a distance $r$ relative to the angular velocity of the rotating local inertial frame, which is dragged at angular velocity $\omega(r)$ :

$$
\bar{\omega}(r)=\Omega-\omega(r) .
$$

The term $e^{-\phi(r)}$ in equation (6) translates $\bar{\omega}(r)$ as measured from infinity to $\bar{\omega}(r)$ as measured in the local inertial frame at $r$. The following auxiliary relations were used to determine values of $\phi(r)$ and $\bar{\omega}(r)$ throughout the star $(r<R)$ in the moment of inertia calculation:

$$
\begin{gathered}
\frac{d \phi(r)}{d r}=-\left[\frac{1}{\rho(r) c^{2}+P(r)}\right] \frac{d P(r)}{d r}, \\
j(r)=\left[1-\frac{2 G m(r)}{r c^{2}}\right] e^{-\phi(r)}, \\
\frac{d}{d r}\left[r^{4} j(r) \frac{d \bar{\omega}(r)}{d r}\right]+4 r^{3} \frac{d j(r)}{d r} \bar{\omega}(r)=0 .
\end{gathered}
$$

An arbitrary value of $\bar{\omega}(0)$ was chosen, and the following boundary conditions imposed in the models:

$$
\begin{gathered}
\frac{d \bar{\omega}(0)}{d r}=0, \\
\bar{\omega}(R)=\Omega-\frac{R}{3} \frac{d \bar{\omega}(R)}{d r} .
\end{gathered}
$$

This latter condition (in which a value for $\Omega$ is determined at the end of the model iteration) was used to scale the arbitrarily chosen $\bar{\omega}(0)$ in such a way as to yield the proper observed $\Omega$ in a subsequent modeling pass. This subsequent pass produced the correct $\bar{\omega}(r)$ for all $r<R$. The condition of finite $\phi(0)$ was also imposed.

Milne's centered algorithm (Harrison et al. 1965) was used for the iteration. Since there is a transition region between the superfluid core and the rigid crust that consists of a mixture of nuclei and free baryons, there is no clear division where the core-crust transition occurs. We chose a transition density near the nuclear density $\left(\rho_{\text {transition }} \sim\right.$ $2.4 \times 10^{14} \mathrm{~g} \mathrm{~cm}^{-3}$ ) in order to separate the two components in our models. From this we obtained $I_{\text {core }} \equiv I\left(r_{\text {transition }}\right)$ and $I_{\text {total }} \equiv I(R)$ for each model NS and could compare the ratio of these with the measured values of $Q$ from glitch observations. In previous work, some authors (e.g., Datta \& Alpar 1993; Shapiro \& Teukolsky 1983) have assumed a slightly lower core-crust transition density than the one used here. This lower transition density reduces the size of the crust, thereby increasing the fractional moment of inertia of the core. Although slight differences in the choice of the transition density do not affect our model results, a significant reduction would lead to a more stringent test of the starquake model (i.e., it would further limit the range of observed $Q$ that would be consistent with the predictions of the starquake model). Thus, our choice of $\rho_{\text {transition }}$ is a conservative one for this purpose.

For angular rotation speeds comparable to those of the Crab and Vela pulsars $\left(\Omega \sim 190\right.$ and $70 \mathrm{rad} \mathrm{s}^{-1}$, respectively), the macroscopic star parameters are affected by first-order rotation effects, but not significantly so by second-order effects.

The Lense-Thirring frame-dragging effect is a firstorder effect that scales with rotational speed as $\Omega / \Omega_{c}$ (Arnett \& Bowers 1977), where $\Omega_{c}=\left(G M / R^{3}\right)^{1 / 2}$, the critical value above which centrifugal mass shedding would occur for the rotating star. For realistic NS models, $\Omega_{c} \sim 13,000 \mathrm{rad} \mathrm{s}^{-1}$. This first-order effect is therefore $\sim 1 \%-2 \%$ for the Crab and Vela pulsars and is taken into consideration in the calculation of the relativistic moment of inertia in the models.

NS mass shifts and deformations in sphericity from rotation (which would complicate the simple spherical stellar modeling presented here) are second-order effects, scaling as $\left(\Omega / \Omega_{c}\right)^{2}$ (Arnett \& Bowers 1977). This term would be significant for millisecond pulsars, but for the Crab pulsar, the second-order effect is $\sim 10^{-4}$, and it is even lower for Vela. Thus, the second-order effect can be safely ignored when considering the macroscopic parameters of our NS models.

\section{RESULTS}

We have produced a set of parameterized NS models using the seven high-density EOSs described above. In order to decide which models in each series were appropriate to use in the comparison with the observed values of $Q$, we used a realistic NS mass range as a reasonable constraint. Models with an NS mass outside of the range were not used in the comparison.

Thorsett \& Chakrabarty (1999) have presented a statistical study of NS masses for the known binary radio pulsar population for which useful mass constraints can be derived. They find that, statistically, the distribution of 


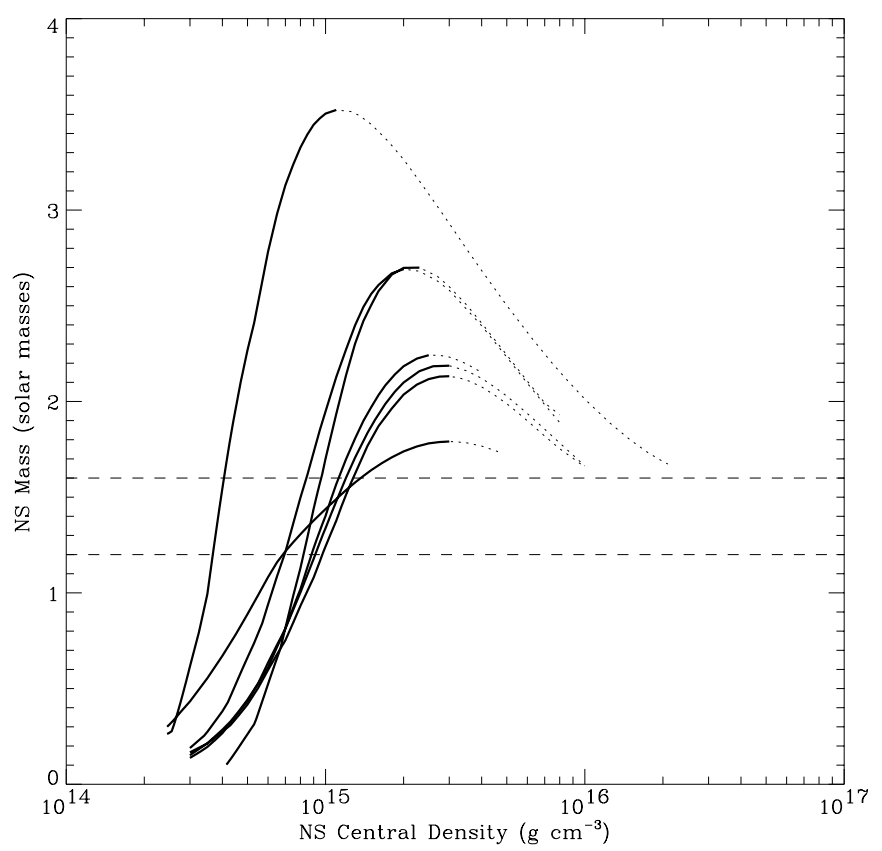

FIG. 1.-Mass vs. central density for NS models constructed from seven high-density EOSs. The seven parameter curves correspond to these seven EOSs in order of increasing maximum mass: GWM, BJW, FPS, WFF, APR(p), APR(s), and HKP. Branches with positive slope (a necessary condition for stability) are indicated with solid lines. The dashed horizontal lines correspond to the mass range $1.4 \pm 0.2 M_{\odot}$, which is assumed to be a realistic NS mass range.

pulsar masses falls in the range $M=1.35 \pm 0.04 M_{\odot}$. This is consistent with the initial NS mass function from simulations of supernovae, which predict that NSs are formed with $M \gtrsim 1.2 M_{\odot}$ (Timmes, Woosley, \& Weaver 1996). For our comparison and analysis, we consider only the mass range $M=1.4 \pm 0.2 M_{\odot}$, which we assume to be a conservative estimate of the realistic NS mass range.

Figure 1 shows NS mass $M \equiv m(R)$ as a function of central density $\rho_{c} \equiv \rho(0)$ for our models constructed using the seven different EOSs described above. NS branches with $d M / d \rho_{c}>0$ satisfy a necessary stability criterion (e.g., Arnett \& Bowers 1977) and are indicated with solid lines. For all EOSs used, there are stable model stars in the mass range $1.4 \pm 0.2 M_{\odot}$. Figure 2 shows NS mass $M$ as function of radius $R$ for the seven EOSs used. Stable NS branches are again indicated with solid lines. None of the models in the stable branches violate the required stability condition $M / R<4 c^{2} / 9 G$ for static relativistic stars (Glendenning 2000).

Figure 3 shows the ratio of the moment of inertia of the core, $I_{\text {core }}$, to the total moment of inertia, $I_{\text {total }}$, as a function of NS mass $M$. For stable models in the mass range of interest $\left(1.4 \pm 0.2 M_{\odot}\right)$, this ratio is confined to $I_{\text {core }} / I_{\text {total }} \gtrsim 0.55$. Starquakes cannot be responsible for producing glitches in a pulsar with measured values of $Q$ consistently outside this range unless the pulsar mass is significantly smaller than expected.

Glitch measurements (including measurements of $Q$ ) for the Crab and Vela pulsars have been published in various places in the literature (see Table 1 for references). However, no comprehensive current listing of all measured values of $Q$ for Crab and Vela glitches exists. Table 1 is a compilation of all measured values of $Q$ published to date for glitches

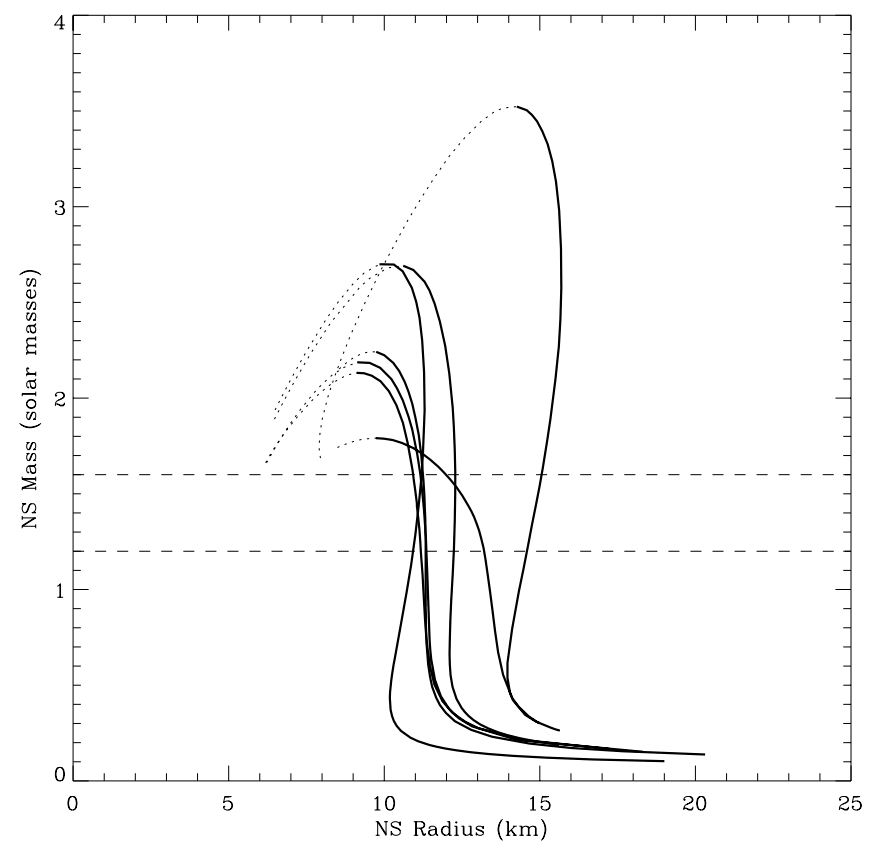

FIG. 2.-Mass vs. radius for NS models constructed from seven highdensity EOSs. Stable branches are indicated with solid lines. The dashed horizontal lines correspond to the mass range $1.4 \pm 0.2 M_{\odot}$, which is assumed to be a realistic NS mass range.

from the Crab and Vela pulsars. Some are different measurements of the same glitch. This complete set of measurements can be used to derive a range of observed $Q$ for each pulsar. From the 21 measurements of $Q$ for $\mathrm{Crab}$

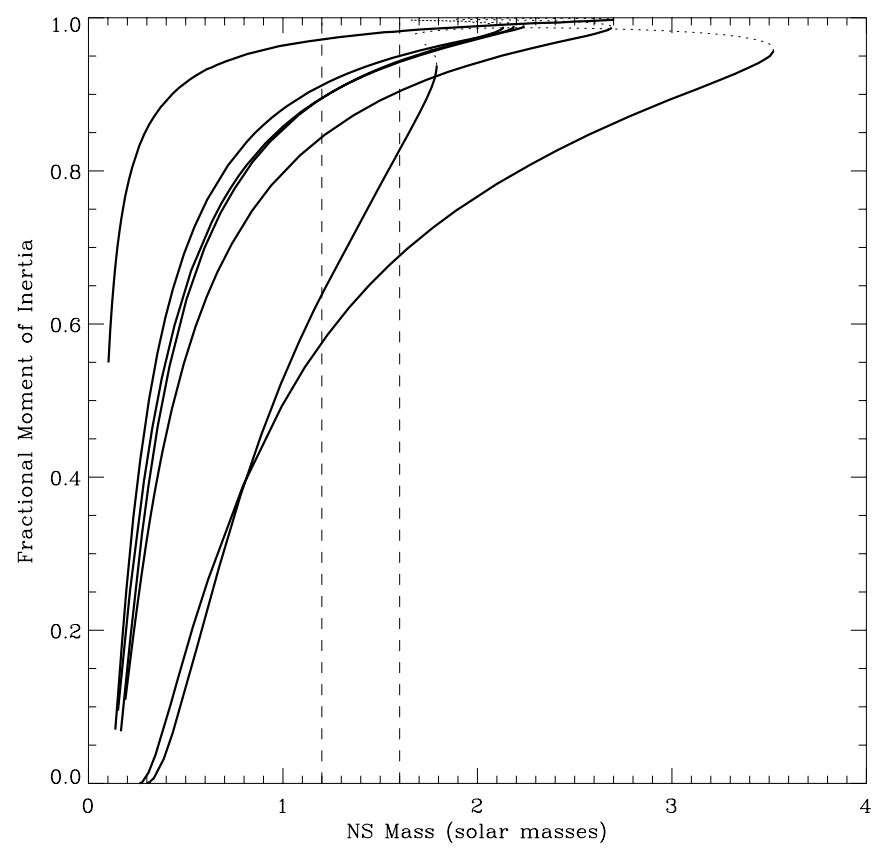

FIG. 3.-Fractional core moment of inertia vs. mass for NS models constructed from seven high-density EOSs. The assumed realistic NS mass range $1.4 \pm 0.2 M_{\odot}$ is indicated by the dashed vertical lines. Stable NS configurations (solid lines) in the mass range $1.4 \pm 0.2 M_{\odot}$ have corresponding $I_{\text {core }} / I_{\text {total }} \gtrsim 0.55$ in all cases. This ratio is consistent with the large values of the glitch healing parameter $Q$ predicted by the starquake model for Crab glitches $(Q \gtrsim 0.7)$, supporting the starquake glitch interpretation. The ratio is inconsistent with the much lower values of $Q$ seen for Vela $(Q \lesssim 0.2)$, indicating that starquakes do not account for Vela glitches. 
TABLE 1

Measured Values of $Q$ for Crab and Vela Glitches

\begin{tabular}{|c|c|c|}
\hline MJD of Glitch & $Q$ & Reference \\
\hline \multicolumn{3}{|c|}{ Crab (PSR B0531+21) } \\
\hline \multirow[t]{4}{*}{$40493 \ldots \ldots \ldots \ldots \ldots \ldots \ldots \ldots \ldots$} & $0.923 \pm 0.073$ & 1 \\
\hline & $0.93 \pm 0.05$ & 2 \\
\hline & $0.94 \pm 0.01$ & 3 \\
\hline & 0.58 & 4 \\
\hline $41163 \ldots \ldots \ldots \ldots \ldots \ldots \ldots \ldots \ldots$ & $0.92 \pm 0.02$ & 3 \\
\hline \multirow{2}{*}{$41250 \ldots \ldots \ldots \ldots \ldots \ldots \ldots \ldots$} & 0.96 (fixed) & 2 \\
\hline & $0.71 \pm 0.02$ & 3 \\
\hline \multirow[t]{3}{*}{42447 …........................ } & $0.96 \pm 0.03$ & 2 \\
\hline & 0.77 & 4 \\
\hline & $0.707 \pm 0.002$ & 3 \\
\hline $43023 .$. & 0.7 (fixed) & 3 \\
\hline $43768 \ldots \ldots \ldots \ldots \ldots \ldots \ldots \ldots \ldots$ & 0.7 (fixed) & 3 \\
\hline 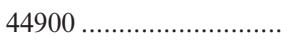 & $\ldots$ & 4 \\
\hline $46664 \ldots \ldots \ldots \ldots \ldots \ldots \ldots \ldots \ldots$ & 1.00 & 4 \\
\hline $47767 \ldots \ldots \ldots \ldots \ldots \ldots \ldots \ldots \ldots$ & 0.89 & 4 \\
\hline 48947 ................................... & 0.87 & 5 \\
\hline 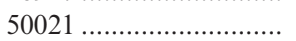 & 0.80 & 5 \\
\hline $50260 \ldots \ldots \ldots \ldots \ldots \ldots \ldots \ldots \ldots$ & 0.68 & 5 \\
\hline 50459 & 0.87 & 5 \\
\hline $50489 \ldots \ldots \ldots \ldots \ldots \ldots \ldots \ldots \ldots$ & $\ldots$ & 5 \\
\hline 50813 & 0.92 & 5 \\
\hline 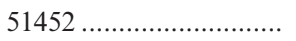 & 0.83 & 5 \\
\hline $51741 \ldots \ldots \ldots \ldots \ldots \ldots \ldots \ldots \ldots$ & $0.80 \pm 0.4$ & 6 \\
\hline \multicolumn{3}{|c|}{ Vela (PSR B0833-45) } \\
\hline $40280 \ldots \ldots .$. & $0.034 \pm 0.01$ & 7 \\
\hline $41192 \ldots \ldots \ldots \ldots \ldots \ldots \ldots \ldots \ldots$ & $0.035 \pm 0.001$ & 7 \\
\hline 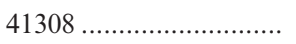 & $0.55 \pm 0.21$ & 7 \\
\hline \multirow[t]{2}{*}{$42683 \ldots \ldots \ldots \ldots \ldots \ldots \ldots \ldots$} & $0.088 \pm 0.008$ & 7 \\
\hline & $0.323 \pm 0.012$ & 8 \\
\hline \multirow[t]{2}{*}{$43681 \ldots \ldots \ldots \ldots \ldots \ldots \ldots \ldots \ldots$} & $0.024 \pm 0.005$ & 7 \\
\hline & $0.220 \pm 0.036$ & 8 \\
\hline $44888 \ldots \ldots \ldots$ & $0.177 \pm 0.001$ & 9 \\
\hline 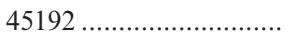 & $0.044 \pm 0.003$ & 10 \\
\hline 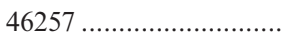 & $0.158 \pm 0.001$ & 10 \\
\hline $47520 \ldots \ldots \ldots \ldots \ldots \ldots \ldots \ldots$ & $\ldots$ & 11 \\
\hline 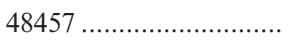 & $\ldots$ & 11 \\
\hline  & $\ldots$ & 12 \\
\hline 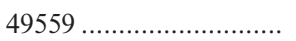 & $\ldots$ & 11 \\
\hline 49591 ................................... & $\ldots$ & 11 \\
\hline 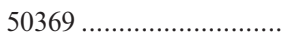 & $0.38 \pm 0.02$ & 12 \\
\hline  & $\ldots$ & 13 \\
\hline
\end{tabular}

REFERENCES.- (1) Boynton et al. 1972; (2) Löhsen 1975; (3) Löhsen 1981; (4) Lyne, Pritchard, \& Smith 1993;

(5) Wong, Backer, \& Lyne 2001; (6) Wang et al. 2001; (7) Downs 1981; (8) Manchester et al. 1983; (9) McCulloch et al. 1983; (10) McCulloch et al. 1987; (11) Lyne et al. 2000; (12) Wang et al. 2000; (13) Dodson, McCulloch, \& Lewis 2002.

glitches, a weighted mean of the values yields $Q=0.72 \pm 0.05 .^{1}$ This is only slightly smaller than the unweighted mean of $Q=0.83$. A range of $Q \gtrsim 0.7$ encompasses the observed distribution for the Crab pulsar. A weighted mean of the 11 measurements of $Q$ for Vela glitches yields $0.12 \pm 0.07$, while an unweighted mean gives $Q=0.18$. All estimates for Vela agree that $Q$ is small, with a likely range of $Q \lesssim 0.2$.

\footnotetext{
${ }^{1}$ For measurements of $Q$ without a quoted uncertainty, an uncertainty of 0.1 was simply assumed in the calculation of the weighted mean.
}

\section{DISCUSSION}

The glitch behavior of the Crab (Alpar et al. 1994, 1996) and Vela (Alpar et al. 1993; Chau et al. 1993) pulsars has been extensively studied previously. Below we discuss and compare our model results with the observed range of $Q$ in the context of this previous work.

\subsection{Vela}

Alpar et al. (1993) and Chau et al. (1993) have found that their model predictions of vortex unpinning in Vela are completely consistent with the observed Vela glitch characteristics. They deduce that the fractional crustal moment of inertia must be greater than $2.6 \%$ for Vela (Chau et al. 1993). Datta \& Alpar (1993) have used a corresponding lower limit of $3.4 \%$ for Vela in an attempt to rule out soft EOSs. Link, Epstein, \& Lattimer (1999) employ a lower limit of $1.4 \%$ for a similar purpose. These estimates imply an upper limit to the fractional core moment of inertia of $\sim 0.98$, which tends to disfavor APR(s) models with mass $M \gtrsim 1.5 M_{\odot}$ but cannot independently constrain the Vela mass, since this condition is satisfied for a wide range of model masses (see Fig. 3). The conclusions made by Alpar et al. (1993) and Chau et al. (1993) that the Vela mass is probably less than $1.4 M_{\odot}$ (and is more likely closer to 1.2 $M_{\odot}$ ) cannot be confirmed or ruled out. Lyne (1992) explains one reason why starquakes cannot be the glitch mechanism for Vela: the required oblateness is not sustainable given the large size of Vela glitches $\left(\Delta \Omega / \Omega \sim 10^{-6}\right)$. After $\sim 100 \mathrm{yr}$, the oblateness would reach zero, and therefore glitches could only have been sustained for $\sim 1 \%$ of the current Vela age. The time interval between Vela glitches is also inconsistent with the much longer intervals predicted by the starquake model (Alpar et al. 1996) unless a solid core model is invoked for Vela (e.g., Canuto \& Chitre 1973; Pines et al. 1974). Starquakes would also produce a thermal energy dissipation during the large Vela glitches that is expected to be observable as a change in X-ray luminosity soon after the glitch occurs. X-ray observations of Vela show no such signal down to limits of less than a few percent in the fractional change in flux (Seward et al. 2000; Helfand, Gotthelf, \& Halpern 2001). The vortex unpinning model does not have this energy dissipation problem for Vela (Alpar 1995). The low values of $Q$ measured for Vela glitches are additional evidence that the predictions of the starquake model do not match observations; otherwise, the implied Vela mass would be too low ( $M \lesssim 0.5 M_{\odot}$ for $Q \lesssim 0.2$ in our models). Our model results confirm previous conclusions that starquakes cannot be a feasible glitch mechanism for the Vela pulsar.

\section{2. $\mathrm{Crab}$}

Alpar et al. (1994, 1996) have studied the behavior of Crab glitches and have determined that the starquake model is at least partially responsible for them. The magnitudes of Crab glitches, combined with the observed glitch rate, support this notion. Lyne (1992) indicates that the small size of Crab glitches $\left(\Delta \Omega / \Omega \sim 10^{-8}\right)$ allows for small changes in oblateness that would not significantly deplete the oblateness over the current lifetime of the Crab. Link, Franco, \& Epstein (1998) and Franco, Link, \& Epstein (2000) also show that the permanent postglitch offsets in the period derivative seen for the Crab pulsar can be accounted for by starquakes: the net torque on the star increases through 
shearing effects. Our model results are also consistent with the starquake interpretation for Crab glitches. The fractional moment of inertia values in our models are comparable to the observed $Q$-values for Crab glitches $(Q \gtrsim 0.7)$ if $M \gtrsim 0.15 M_{\odot}$, which seems likely. Alpar et al. (1994) set a lower limit of $0.2 \%$ for the fractional crustal moment of inertia. This is not constraining our models, since the corresponding fractional core moment of inertia range $I_{\text {core }} / I_{\text {total }} \lesssim 0.998$ is easily satisfied. Our results support previous suggestions that starquakes could be responsible for Crab glitches.

\section{CONCLUSIONS}

Using parameterized NS models produced from seven representative EOSs of superdense matter, we find that the fractional moment of inertia of the core component of the model stars is $I_{\text {core }} / I_{\text {total }} \gtrsim 0.55$ for all stable configurations in the assumed realistic NS mass range of $1.4 \pm 0.2 M_{\odot}$ (Fig. $3)$. This ratio, which is predicted by the starquake model to equal the glitch healing parameter $Q$, is not consistent with the observed range $Q \lesssim 0.2$ for Vela glitches (see Table 1), unless the Vela pulsar mass is unrealistically small $(M \lesssim 0.5$ $\left.M_{\odot}\right)$. This confirms results from previous studies of the Vela pulsar that indicate that starquakes are not the cause of Vela glitches (Lyne 1992; Alpar et al. 1993; Chau et al. 1993; Alpar 1995). The much larger values of $Q \gtrsim 0.7$ seen for Crab glitches (Table 1) are consistent with the moment of inertia values of our models for realistic masses, as predicted by the starquake model. These results support previous conclusions from the analysis of Crab glitch behavior (Alpar et al. 1994, 1996; Link et al. 1998; Franco et al. 2000) in which starquakes have been proposed as the Crab glitch mechanism. Repeated and accurate measurements of $Q$ for other glitching pulsars in the future (if obtainable) could be used as a simple test of the starquake glitch model and may help resolve whether differences between Crab-like and Vela-like glitches can be understood on evolutionary grounds (e.g., Alpar 1995).

We thank the referee for helpful comments that improved the manuscript and for suggesting the inclusion of additional EOSs in the modeling. M. D. was partially supported by a grant of the Polish Committee for Scientific Research.

Akmal, A., Pandharipande, V. R., \& Ravenhall, D. G. 1998, Phys. Rev. C, REFERENCES

58,1804

Alpar, M. A. 1995, in The Lives of Neutron Stars, ed. M. A. Alpar, U. Kízíloğlu, \& J. van Paradijs (Dordrecht: Kluwer), 185

Alpar, M. A., Anderson, P. W., Pines, D., \& Shaham, J. 1984, ApJ, 278, 791

Alpar, M. A., Chau, H. F., Cheng, K. S., \& Pines, D. 1993, ApJ, 409, 345 1994, ApJ, 427, L29

1996, ApJ 459, 706

Anderson, P. W., \& Itoh, N. 1975, Nature, 256, 25

Arnett, W. D., \& Bowers, R. L. 1977, ApJS, 33, 415

Baym, G., Bethe, H., \& Pethick, C. 1971, Nucl. Phys. A, 175, 225

Baym, G., Pethick, C., \& Sutherland, P. 1971, ApJ, 170, 299

Baym, G., \& Pines, D. 1971, Ann. Phys., 66, 816

Bethe, H., \& Johnson, M. 1974, Nucl. Phys. A, 230, 1

Boynton, P. E., Groth, E. J., Hutchinson, D. P., Nanos, G. P., Partridge,

R. B., \& Wilkinson, D. T. 1972, ApJ, 175, 217

Canuto, V. 1975, ARA\&A, 13, 335

Canuto, V., \& Chitre, S. M. 1973, Nature Phys. Sci., 243, 63

Chau, H. F., McCulloch, P. M., Nandkumar, R., \& Pines, D. 1993, ApJ, 413, L113

Datta, B., \& Alpar, M. A. 1993, A\&A, 275, 210

Dodson, R. G., McCulloch, P. M., \& Lewis, D. R. 2002, ApJ, 564, L85

Downs, G. S. 1981, ApJ, 249, 687

Feynman, R. P., Metropolis, N., \& Teller, E. 1949, Phys. Rev., 75, 1561

Forest, J. L., Pandharipande, V. R., \& Friar, J. L. 1995, Phys. Rev. C, 52, 568

Franco, L. M. Link, B. \& Epstein, R. I. 2000, ApJ, 543, 987

Friedman, B., \& Pandharipande, V. R. 1981, Nucl. Phys. A, 361, 502

Glendenning, N. K. 2000, Compact Stars: Nuclear Physics, Particle Physics, and General Relativity (New York: Springer)

Glendenning, N. K., Weber, F., \& Moszkowski, S. A. 1992, Phys. Rev. C, 45,844

Gold, T. 1968, Nature, 218, 731 1969, Nature, 221, 25

Haensel, P. Kutschera, M., \& Proszynski, M. 1981, A\&A, 102, 299

Harrison, B. K., Thorne, K. S., Wakano, M., \& Wheeler, J. A. 1965 , Gravitation Theory and Gravitational Collapse (Chicago: Univ. Chicago Press)

Helfand, D. J., Gotthelf, E. V., \& Halpern, J. P. 2001, ApJ, 556, 380

Hewish, A., Bell, J. S., Pilkington, P. F., Scott, P. F., \& Collins, R. A. 1968, Nature, 217, 709

Lagaris, I. E. \& Pandharipande, V. R. 1981a, Nucl. Phys. A, 359, 331 1981b, Nucl. Phys. A, 359, 349
Link, B., Epstein, R. I., \& Lattimer, J. M. 1999, Phys. Rev. Lett., 83, 3362

Link, B., Franco, L. M., \& Epstein, R. I. 1998, ApJ, 508, 838

Löhsen, E. 1975, Nature, 258, 688

Löhsen, E. H. G. 1981, A\&AS, 44, 1

Lorenz, C. P., Ravenhall, D. G., \& Pethick, C. J. 1993, Phys. Rev. Lett., 70, 379

Lyne, A. G. 1992, Philos. Trans. R. Soc. London A, 341, 29

Lyne, A. G., Pritchard, R. S., \& Smith, F. G. 1993, MNRAS, 265, 1003

Lyne, A. G., Shemar, S. L., \& Smith, F. G. 2000, MNRAS, 315, 534

Manchester, R. N., Newton, L. M., Goss, W. M., \& Hamilton, P. A. 1983, MNRAS, 202, 269

McCulloch, P. M., Hamilton, P. A., Royle, G. W. R., \& Manchester, R. N. 1983, Nature, 302, 319

McCulloch, P. M., Klekociuk, A. R., Hamilton, P. A., \& Royle, G. W. R. 1987, Australian J. Phys., 40, 725

Oppenheimer, J. R., \& Volkoff, G. M. 1939, Phys. Rev., 55, 374

Ostriker, J. P., \& Gunn, J. E. 1969, ApJ, 157, 1395

Pines, D., Shaham, J., \& Ruderman, M. A. 1974, IAU Symp. 53, Physics of Dense Matter, ed. C. J. Hansen (Dordrecht: Reidel), 189

Pudliner, B. S., Pandharipande, V. R., Carlson, J., \& Wiringa, R. B. 1995, Phys. Rev. Lett., 74, 4396

Radhakrishnan, V., \& Manchester, R. N. 1969, Nature, 222, 228

Reichley, P. E., \& Downs, G. S. 1969, Nature, 222, 229

Reid, R. V. 1968, Ann. Phys., 50, 411

Ruderman, M. 1972, ARA\&A, 10, 427

. 1976, ApJ, 203, 213

Serot, B. D. 1979, Ph.D. thesis, Stanford Univ.

Seward, F. D., Alpar, M. A., Flanagan, C. Kízíloğlu, Ü., Markwardt, C., McCulloch, P., \& Ogelman, H. 2000, ApJ, 536, 948

Shapiro, S. L., \& Teukolsky, S. A. 1983, Black Holes, White Dwarfs, and Neutron Stars: The Physics of Compact Objects (New York: Wiley)

Shemar, S. L., \& Lyne, A. G. 1996, MNRAS, 282, 677

Skyrme, T. 1959, Nucl. Phys., 9, 665

Thorsett, S. E., \& Chakrabarty, D. 1999, ApJ, 512, 288

Timmes, F. X., Woosley, S. E., \& Weaver, T. A. 1996, ApJ, 457, 834

Walecka, J. 1974, Ann. Phys., 83, 491

Wang, N., Manchester, R. N., Pace, R. T., Bailes, M., Kaspi, V. M., Stappers, B. W., \& Lyne, A. G. 2000, MNRAS, 317, 843

Wang, N., Wu, X., Manchester, R. N., Zhang, J., Lyne, A. G., \& Yusup, A. 2001, Chinese J. Astron. Astrophys, 1, 195

Wiringa, R. B., Fiks, V., \& Fabrocini, A. 1988, Phys. Rev. C, 38, 1010

Wiringa, R. B., Stoks, V. G. J., \& Schiavilla, R. 1995, Phys. Rev. C, 51, 38

Wong, T., Backer, D. C., \& Lyne, A. G. 2001, ApJ, 548, 447

Zimanyi, J., \& Moszkowski, S. A. 1990, Phys. Rev. C, 42, 1416 